\title{
Osteosarcopenia and trabecular bone score in patients with type 2 diabetes mellitus
}

Luciana Muniz Pechmann'

https://orcid. org/0000-0002-2965-5268

Ricardo R. Petterle 2

https://orcid. org/0000-0001-7735-1077

Carolina A. Moreira ${ }^{3}$

https://orcid.org/0000-0002-9908-4907

Victoria Z. C. Borba ${ }^{3}$

https://orcid.org/0000-0003-0555-0880

\begin{abstract}
Objective: To evaluate the prevalence of osteosarcopenia and the association of osteosarcopenia with trabecular bone score (TBS) in a group of patients with type 2 diabetes mellitus(T2DMG) compared with a paired control group (CG). Materials and methods: Cross-sectional study with men and women $\geq 50$ years recruited by convenience. Patients in both groups answered questionnaires and underwent evaluation of bone mineral density (BMD), handgrip strength (HGS), and TBS. The T2DMG also underwent a gait speed (GS) test. Sarcopenia was defined as low lean mass plus low HGS or GS according to the Foundation for the National Institute of Health Sarcopenia Project, and osteosarcopenia was deemed present when sarcopenia was associated with osteopenia, osteoporosis, or low-energy trauma fractures. Results: The T2DMG $(n=177)$ and CG $(n=146)$ had, respectively, mean ages of $65.1 \pm 8.2$ years and $68.8 \pm 11.0$ years and $114(64.4 \%)$ and $80(54.7 \%)$ women.T2DMG versus the CG had higher rates of osteosarcopenia (11.9\% versus $2.14 \%$, respectively, $p=0.010)$, sarcopenia $(12.9 \%$ versus $5.4 \%$, respectively, $p<0.030)$, and fractures $(29.9 \%$ versus $18.5 \%$, respectively, $p=0.019)$, and lower HGS values $(24.4 \pm 10.3 \mathrm{~kg}$ versus $30.9 \pm 9.15 \mathrm{~kg}$, respectively, $p<0.001$ ), but comparable BMD values. Mean TBS values were $1.272 \pm 0.11$ and $1.320 \pm 0.12$, respectively $(p=0.001)$. On multivariate analysis, age, greater waist circumference, fractures, and osteoporosis increased the risk of degraded TBS. Osteosarcopenia was associated with diabetes complications $(p=0.03)$, calcium and vitamin $D$ supplementation $(p=0.01)$, and all components of osteosarcopenia diagnosis $(p<0.05)$. Conclusion: Compared with the CG, the T2DMG had a higher prevalence of osteosarcopenia, sarcopenia, and fractures and lower bone quality assessed by TBS. Arch Endocrinol Metab. 2021;65(6):801-10
\end{abstract}

Keywords

Sarcopenia; type 2 diabetes mellitus; muscle weakness; fractures; osteoporosis

\author{
1 Divisão de Endocrinologia (Serviço \\ de Endocrinologia e Metabologia \\ do Paraná - SEMPR), Hospital de \\ Clínicas da Universidade Federal \\ do Paraná e Centro de Diabetes \\ Curitiba, Curitiba, PR, Brasil \\ ${ }^{2}$ Setor de Ciências da Saúde, \\ Faculdade de Medicina, \\ Universidade Federal do \\ Paraná, Curitiba, PR, Brasil \\ ${ }^{3}$ Divisão de Endocrinologia (Serviço \\ de Endocrinologia e Metabologia \\ do Paraná - SEMPR), Hospital \\ de Clínicas, Universidade Federal \\ do Paraná, Curitiba, PR, Brasil
}

\section{INTRODUCTION}

$\mathrm{T}$ ype 2 diabetes mellitus (T2DM) affects bone and muscle health. Osteoporosis, fractures, sarcopenia, and T2DM are common in the elderly population, and recent evidence has indicated common pathophysiological pathways linking T2DM to osteoporosis and sarcopenia (1). Bone, muscle, and glucose metabolism are intimately intertwined in a complex network (2). Indeed, patients with T2DM with poor glycemic control and chronic complications have an increased risk of fractures, despite greater bone mineral density (BMD) compared with individuals without T2DM $(3,4)$.

This apparent contradiction of increased BMD associated with a greater risk of fracture in patients with
T2DM is probably due to poor bone quality undetected by BMD measurement $(4,5)$. This observation highlights the importance of assessing bone strength and fracture risk with methods other than BMD, such as trabecular bone score (TBS), an indirect measure of bone quality (6). Indeed, low TBS values have been associated with fractures in patients with T2DM (7-9). Additionally, insulin appears to affect bone quality and quantity in patients with T2DM. Hyperinsulinemia associated with insulin resistance and visceral obesity - all frequent in patients with T2DM - correlate with increased bone mass but deteriorated bone quality and muscle strength and loss of muscle function (10).

In terms of muscle quality and function, sarcopenia occurs earlier and 3-16 times more frequently in individuals 
with diabetes compared with those without diabetes. Additionally, patients with sarcopenia and T2DM are predisposed to long-term diabetes complications, frailty, hospitalizations, and premature death $(10,11)$.

The link between sarcopenia and osteoporosis remains elusive, owing mostly to lack of a standardized definition for sarcopenia and adoption of different criteria defining this condition across studies $(12,13)$. Still, sarcopenia and osteoporosis coexist in many individuals, a condition known as osteosarcopenia. Compared with patients with sarcopenia or osteoporosis alone, those with osteosarcopenia have worse healthrelated outcomes regarding falls, fractures, physical performance, quality of life, and mortality (13).

Only a few studies to date have assessed the effect of sarcopenia on fractures and bone quality in T2DM, while the impact of comorbidities and chronic complications on the development of osteosarcopenia in this population remains unknown (13-15). Also, the prevalence of this condition in patients with T2DM is still unclear despite the critical impact of osteosarcopenia on health. Based on these considerations, the aims of this study were to investigate the prevalence of osteosarcopenia and the association between osteosarcopenia and bone microarchitecture assessed by TBS in patients with T2DM compared with controls.

\section{MATERIALS AND METHODS}

This was a cross-sectional, controlled study in patients with T2DM treated at the research center of a tertiary hospital. The study protocol was approved by the institution's ethics committee (number 53569116.7.0000.0096).

\section{Study sample and data collection}

Men and women $(\geq 50$ years) with treated T2DM diagnosed for $\geq 1$ year were recruited by convenience during routine visits to the outpatient clinic of Serviço de Endocrinologia e Metabologia do Hospital de Clinicas da Universidade Federal of Paraná (SEMPR). Patients were selected during this visit after inclusion and exclusion criteria were evaluated and comprised the T2DM group [T2DMG]) (Figure 1). Assessments were performed on the day of the medical appointment between March to October 2019.

Community-dwelling healthy volunteers belonging to the SEMPR database with normal gait and without T2DM or glucose intolerance comprised

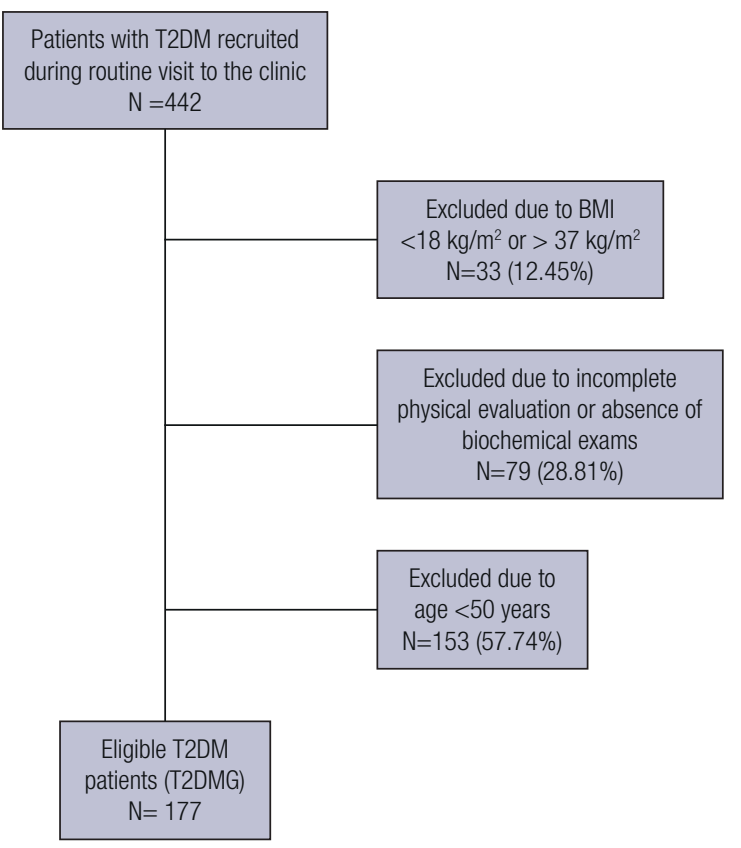

Figure 1. Flowchart of the selection process of patients included in the type 2 diabetes mellitus (T2DM) group.

the control group (CG). CG were matched by age and sex with patients in the T2DMG using the frequency matching method.

The study excluded professional athletes; patients unable to understand the study protocol, immobilized, or disabled; individuals with body mass index (BMI) $>37 \mathrm{~kg} / \mathrm{m}^{2}$ or $<18 \mathrm{~kg} / \mathrm{m}^{2}$; patients with chronic diseases under current treatment that could interfere with the assessment of the variables and those receiving hormone replacement therapy, medications affecting muscle mass or body composition (e.g., diuretics, corticosteroids), and protein supplements.

\section{Questionnaire}

A structured questionnaire developed for the study collected baseline disease characteristics, treatment, comorbidities, lifestyle habits, amount of dietary calcium intake from dairy foods, and a self-reported history of low-energy trauma fractures. Calcium or vitamin D supplementation was evaluated as present or absent. Daily calcium intake was classified based on dietary recommendations (recommended amount > $1,200 \mathrm{mg}$ ) (16). Insulin use and diabetes complications (retinopathy and cataract, distal motor sensory peripheral neuropathy, nephropathy, and cardiovascular disease) were retrieved from medical records and confirmed by the patients' physicians. 


\section{Anthropometric measurements}

Weight was measured using a scale with accuracy of $100 \mathrm{~g}$ and capacity $\leq 150 \mathrm{~kg}$ (Indústrias Filizola SA, São Paulo, Brazil). Height was measured with a Tonelli stadiometer with $0.1 \mathrm{~cm}$ accuracy (IN Tonelli SA, Santa Catarina, Brazil). BMI classification followed the World Health Organization (WHO) recommendations (17). Waist circumference (WC) was obtained in the largest abdominal perimeter between the last rib and the iliac crest, values $\geq 102 \mathrm{~cm}$ and $\geq 88 \mathrm{~cm}$ in men and women, respectively, were considered increased (18).

\section{Evaluation of body composition and BMD}

Body composition (BC) and BMD were assessed using the dual-energy $\mathrm{x}$-ray absorptiometry (DXA) system Lunar Prodigy equipped with the software enCORE, v18 (GE Medical Systems, Madison, WI, USA). Total body composition (BC), lumbar spine (L1-L4), total hip, and femoral neck BMD were analyzed following the International Society for Clinical Densitometry guidelines (19). BC parameters were further analyzed by sex. Precision coefficients were $1.30 \%$ for lumbar spine, $1.28 \%$ for femoral neck, and $0.56 \%$ for total femur. BMD categories were classified according to the WHO criteria (20) as normal, osteopenia, and osteoporosis. DXA was performed by a certified operator, and the results were analyzed by a certified radiologist. The sum of arm and leg lean mass obtained from the $\mathrm{BC}$ analysis yielded the appendicular lean mass (ALM).

\section{Previous nonvertebral fractures}

Self-reported history of low-energy trauma fractures occurring after 50 years of age excluding skull, toes, and face fractures, were categorized as absent or present.

\section{TBS}

TBS was measured from lumbar spine (in Ll-L4 vertebrae not excluded from the BMD measurement) DXA scans using TBS iNsight, v3.0 (MediMaps, Geneva, Switzerland). The values were stratified according to those published in studies including Latin American populations. According to the DXA manufacturer, the microarchitecture was considered degraded with values $\leq 1.230$, partially degraded with values $>1.230$ and $<1.310$, and normal with values $\geq 1.310(21)$.

\section{Muscle mass}

Muscle mass (MM) was calculated as the ratio of ALM to BMI values, and low MM (LMM) was defined as $\mathrm{MM}<0.789$ in men and $<0.512$ in women, according to FNIH criteria. Total lean mass (TLM), obtained in the $\mathrm{BC}$ assessment, was also analyzed according to sex.

\section{Sarcopenia, and osteosarcopenia}

Sarcopenia was defined according to FNIH criteria as low MM associated with low HGS or low physical performance according to the GS and data for this sample already published elsewhere (22). Osteosarcopenia was deemed present in participants with sarcopenia plus low BMD (osteopenia or osteoporosis) or past self- reported low-energy trauma fractures after the age of 50 years (13).

\section{Biochemical evaluation}

Laboratory data from participants in the T2DMG were collected from medical records (the last evaluation was considered when $<3$ months) or from a fasting blood sample collected on the DXA evaluation day. Biochemical evaluation included fasting plasma glucose (normal range $[\mathrm{NR}]<100 \mathrm{mg} / \mathrm{dL}$ ), serum calcium (NR 8.5-10.2 mg/dL), glycated hemoglobin (HbAlc; National Glycohemoglobin Standardization Program, NR $<5.8 \%$ [International Federation of Clinical Chemistry $68 \mathrm{mmol} / \mathrm{mol}])$, and serum parathyroid hormone (NR 15-68 pg/mL). Based on serum 25-hydroxyvitamin D levels measured by immunochemiluminescence (LIAISON, DiaSorin, Saluggia, Italy; CV $\leq 5 \%$ ), the participants were classified as having normal $(\geq 30 \mathrm{ng} / \mathrm{mL})$, deficient $(<20 \mathrm{ng} / \mathrm{mL})$, or insufficient $(21-29 \mathrm{ng} / \mathrm{mL})$ vitamin D levels (23).

\section{Statistical analysis}

All statistical analyses were performed using the $\mathrm{R}$ Core Team, v3.4.4 (R Foundation for Statistical Computing, Vienna, Austria). Normality was tested with the Kolmogorov-Smirnov test. Qualitative data are presented in absolute and relative frequencies and quantitative data as mean \pm standard deviation or median (minimum and maximum) values. Quantitative variables were compared with Student's $t$ or Mann-Whitney test. Comparisons of three or more groups were made with the Kruskal-Wallis test and the post hoc test, while Fisher's exact or chi-square test was used for qualitative analyses. Correlations between parametric variables 
were analyzed with Pearson's correlation. Multivariate logistic regression was performed considering TBS as a dependent variable and as independent variables fractures, osteoporosis, osteopenia, BMI, high WC, low HGS, age, sex, weight, and BMD at all sites. Statistical significance was considered at $\mathrm{p}<0.05$.

\section{RESULTS}

In the T2DMG, 442 patients with T2DM were screened and 265 were excluded, yielding 177 patients in this group as shown in Figure 1 (mean age $65.6 \pm 8.6$ years, 114 women). The CG comprised 146 individuals (mean age $65.0 \pm 9.1$ years, 80 women). More than $70 \%$ of the subjects in each group were older than 60 years. In all, $67.7 \%$ of the patients in the T2DMG were treated with insulin with or without oral agents. The mean dairy calcium intake was below the recommended amount in both groups and was lower in the T2DMG compared with the CG. Participants in the T2DMG compared with those in the CG also had more comorbidities and history of fractures (Table 1). CG had higher glomerular filtration rate and lower BMI than T2DMG.

In the T2DMG, the mean duration of T2DM was $15.4 \pm 8.2$ years, and the mean HbAlc level was $8.5 \pm$ $5.7 \%$. Overall, $66.6 \%$ of the participants in this group had an off-target HbAlc level (>7\%) (24). Hypovitaminosis $\mathrm{D}$ and secondary hyperparathyroidism were observed in $80(45.1 \%)$ and $46(41.4 \%)$ patients, respectively, in the T2DMG. One-third of the T2DMG patients had macrovascular or microvascular complications (Table 1 ).

\section{Nonvertebral fractures}

The T2DMG had more patients with a history of nonvertebral low-energy trauma fractures $(\mathrm{n}=53$, $29.9 \%)$ than the CG $(n=26,18.5 \% ; \mathrm{p}=0.001)$. The most common fracture sites were the legs, forearm, ankle, and wrist. When only patients with a history of fracture in each group were compared, the T2DMG had lower mean BMD values at all sites $(\mathrm{p}<0.001$ for all $)$ and increased rates of osteoporosis $(p=0.003)$, distal motor sensory peripheral neuropathy $(\mathrm{p}=0.043)$, and low HGS ( $\mathrm{p}=0.003)$.

\section{BMD, body composition, and TBS}

Among men, total hip and lumbar spine BMD values and T-scores were greater in the T2DMG compared with the CG $(\mathrm{p}<0.005$ for all), while among women, BMD values at all sites were similar between groups (Figure 3). In addition, the rates of osteopenia and osteoporosis were comparable between groups, although values of BMI and TLM were greater in the T2DMG compared with the CG in both sexes $(\mathrm{p}<0.001)$ (Table 1$)$. Values of ALM were also greater in the T2DMG, but when ALM was corrected for BMI, the difference with the CG was no longer significant (Table 1).

TBS values were measured in 158 and 84 subjects in the T2DMG and CG, respectively. The mean TBS value was lower $(1.272 \pm 0.11)$ in the T2DMG compared with the CG $(1.320 \pm 0.12, \mathrm{p}=0.001)$. Additionally, the T2DMG had more patients with degraded TBS $(\mathrm{p}=0.003)$ (Table 1, Figure 2).

In the T2DMG, the mean TBS was lower in Whites compared with Blacks $(\mathrm{p}=0.016)$ and in patients with increased versus normal WC $(\mathrm{p}=$ $0.035)$, osteopenia $(\mathrm{p}=0.003)$, or osteoporosis ( $\mathrm{p}$ $<0.001$ ) versus normal BMD, with versus without a fracture history $(\mathrm{p}=0.009)$, and with versus without supplementation of calcium, vitamin $\mathrm{D}$, or both ( $\mathrm{p}$ $<0.001$ for all). Of 46 patients in the T2DMG with a history of fracture who had TBS evaluated, 19 $(41.3 \%)$ had degraded TBS $(1.230 \pm 0.108)$ and 14 (30.4\%) had normal BMD.

In the T2DMG, TBS correlated negatively with age $(\mathrm{r}=-0.315, \mathrm{p}<0.001)$ and positively with weight $(\mathrm{r}=$ $0.408, \mathrm{p}<0.001)$, BMI $(\mathrm{r}=0.195, \mathrm{p}=0.014)$, BMD T-scores at all sites (lumbar spine, $\mathrm{r}=0.559, \mathrm{p}<0.001$; femoral neck, $\mathrm{r}=0.531, \mathrm{p}<0.001$; and total hip, $\mathrm{r}=$ $0.564, \mathrm{p}<0.001)$, HGS $(\mathrm{r}=0.400, \mathrm{p}<0.001)$, and GS $(\mathrm{r}=0.273, \mathrm{p}=0.015)$. Mean TBS was associated to osteoporosis and fractures $(\mathrm{p}<0.005)$ in both sexes. TBS had no correlation with other comorbidities or chronic diabetes complications.

On multivariate analysis using mean TBS as a dependent variable, and fractures, osteoporosis, osteopenia, BMI, increased WC, low TLM, low HGS, age, sex, weight, and BMD at all sites as independent variables, age, increased WC, history of fractures, lower TLM, and osteoporosis were associated with a high odds of having degraded TBS. In contrast, weight, male sex, and total hip BMD emerged as factors that confer a lower probability of having degraded TBS (Table 2).

\section{Evaluation of low TBS in T2DMG}

In the T2DMG, 58 (36.7\%) and 98 (62\%) participants had low and normal TBS, respectively. Patients of 
Table 1. Clinical and laboratory characteristics of the study participants

\begin{tabular}{|c|c|c|c|}
\hline & T2DMG $(n=177)$ & $C G(n=146)$ & $P$ values \\
\hline Age (years) & $65.6 \pm 8.6$ & $65.0 \pm 9.1$ & 0.520 \\
\hline Sex & & & 0.070 \\
\hline Women & $114(64.4 \%)$ & $80(54.7 \%)$ & \\
\hline Men & $63(35.5 \%)$ & $66(45.2 \%)$ & \\
\hline \multicolumn{4}{|l|}{ Ethnicity } \\
\hline Caucasians & $149(84.2 \%)$ & $139(95.2 \%)$ & 0.440 \\
\hline Mulattos/Blacks & $26(14.6 \%)$ & $5(3.42 \%)$ & $<0.010$ \\
\hline BMI $\left(\mathrm{kg} / \mathrm{m}^{2}\right)$ & $29.2 \pm 4.89$ & $26.2 \pm 3.10$ & $<0.010$ \\
\hline Waist circumference (cm) & $99.7 \pm 11.0$ & $89.3 \pm 10.0$ & $<0.010$ \\
\hline Menopause & $111(95.6 \%)$ & 69 (86.2\%) & 0.090 \\
\hline Current smoking & $12(6.77 \%)$ & $6(4.10 \%)$ & 0.720 \\
\hline Calcium intake (mg/day) & $403 \pm 3.5$ & $651 \pm 3.8$ & $<0.010$ \\
\hline \multicolumn{4}{|l|}{ Comorbidities } \\
\hline Dyslipidemia & $154(87.0 \%)$ & 35 (27.7\%) & $<0.001$ \\
\hline Hypertension & $145(82.0 \%)$ & $39(30.3 \%)$ & $<0.010$ \\
\hline Hypothyroidism & $50(28.2 \%)$ & $16(12.4 \%)$ & $<0.001$ \\
\hline \multicolumn{4}{|l|}{ Complications } \\
\hline Any complications & $107(60.4 \%)$ & $\mathrm{N} / \mathrm{A}$ & \\
\hline Cardiovascular disease & 53 (29.9\%) & $\mathrm{N} / \mathrm{A}$ & \\
\hline Retinopathy & $52(29.3 \%)$ & $\mathrm{N} / \mathrm{A}$ & \\
\hline Distal motor sensory peripheral neuropathy & $50(28.2 \%)$ & N/A & \\
\hline Nephropathy & $45.0(25.4 \%)$ & N/A & \\
\hline \multicolumn{4}{|l|}{ Biochemical analysis } \\
\hline Creatinine & $1.18 \pm 1.1$ & $0.92 \pm 0.23$ & $<0.001$ \\
\hline Glomerular filtration rate (MDRD) mL/min/1.73 m² & $71.0 \pm 19.0$ & $78.0 \pm 15.0$ & $<0.010$ \\
\hline Calcium mg/dL & $9.41 \pm 0.9$ & $9.38 \pm 0.47$ & $<0.001$ \\
\hline PTH pg/mL & $72.7 \pm 50.5$ & $N / A$ & $\mathrm{~N} / \mathrm{A}$ \\
\hline 25-hydroxyvitamin D (ng/mL) & $29.6 \pm 13.3$ & $\mathrm{~N} / \mathrm{A}$ & $\mathrm{N} / \mathrm{A}$ \\
\hline Deficient (<20 ng/mL) & $80(45.1 \%)$ & & \\
\hline Insufficient (21 a 29 ng/mL) & $47(26.6 \%)$ & & \\
\hline Sufficient ( $\geq 30 \mathrm{ng} / \mathrm{mL}$ ) & $50(28.2 \%)$ & & \\
\hline $\mathrm{HbA1c}$ & $8.5 \% \pm 5.7$ & $\mathrm{~N} / \mathrm{A}$ & $\mathrm{N} / \mathrm{A}$ \\
\hline \multicolumn{4}{|l|}{ Treatment of diabetes } \\
\hline Oral agent & $56(31.5 \%)$ & $\mathrm{N} / \mathrm{A}$ & N/A \\
\hline Insulin only & $22(12.4 \%)$ & N/A & $\mathrm{N} / \mathrm{A}$ \\
\hline Insulin plus oral agent & $98(55.3 \%)$ & $\mathrm{N} / \mathrm{A}$ & $\mathrm{N} / \mathrm{A}$ \\
\hline History of fracture & 53 (29.9\%) & 26 (18.5\%) & 0.001 \\
\hline ALM (kg) & $20.2 \pm 6.7$ & $18.0 \pm 4.1$ & 0.001 \\
\hline ALM/IMC & 0.70 & 0.69 & 0.810 \\
\hline TBS & $1.272 \pm 0.11$ & $1.320 \pm 0.12$ & 0.001 \\
\hline
\end{tabular}

Data are presented as mean \pm standard deviation or frequency (percentage). T2DMG: type 2 diabetes mellitus group; CG: control group; BMI: body mass index; PTH: parathyroid hormone; HbA1c: glycated hemoglobin; TBS: trabecular bone score; TLM: total lean mass; ALM: appendicular lean mass; N/A: not available. P values < 0.05 were considered significant.

both sexes with low TBS (partially and degraded, n $=98,62.0 \%)$ compared with those with normal TBS $(\mathrm{n}=58,36.7 \%)$ were older $(67.1 \pm 9.0$ years versus $63.1 \pm 7.3$ years, respectively, $\mathrm{p}<0.005)$, more likely to have a history of fracture $(\mathrm{n}=37,38.9 \%$ versus $\mathrm{n}$ $=10,16.1 \% ; \mathrm{p}=0.004)$, a diagnosis of osteoporosis, and had lower BMD at all sites $(\mathrm{p}<0.005)$. Men with low TBS had more frequently cataract $(\mathrm{p}=0.029)$ and tended to have higher fasting glucose levels $(\mathrm{p}=0.06)$ than those with normal TBS, while women with low TBS had higher PTH levels $(\mathrm{p}=$ 0.018 ) and took calcium and vitamin D supplements $(\mathrm{p}=0.017)$ more frequently than those with normal TBS (Supplementary Table). 


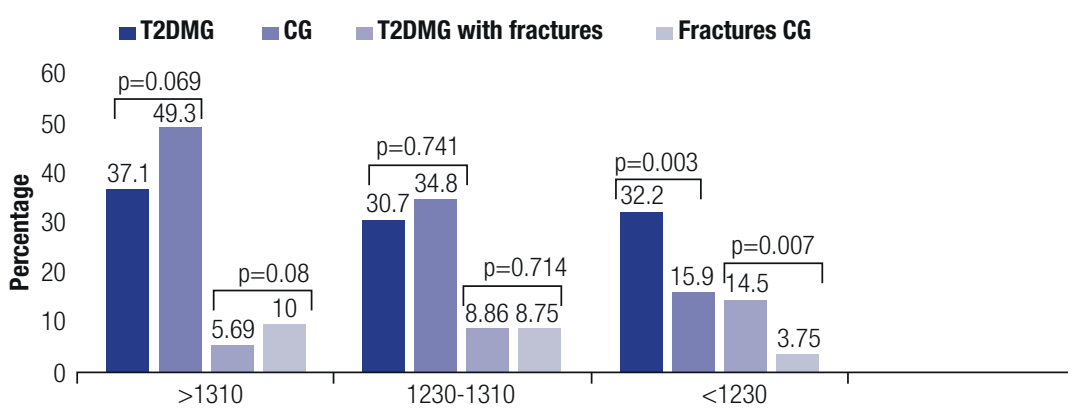

Trabecular Bone Score

Figure 2. Trabecular bone score in T2DM patients and controls with and without a history of fractures.

T2DMG: type 2 diabetes mellitus group; CG: control group.

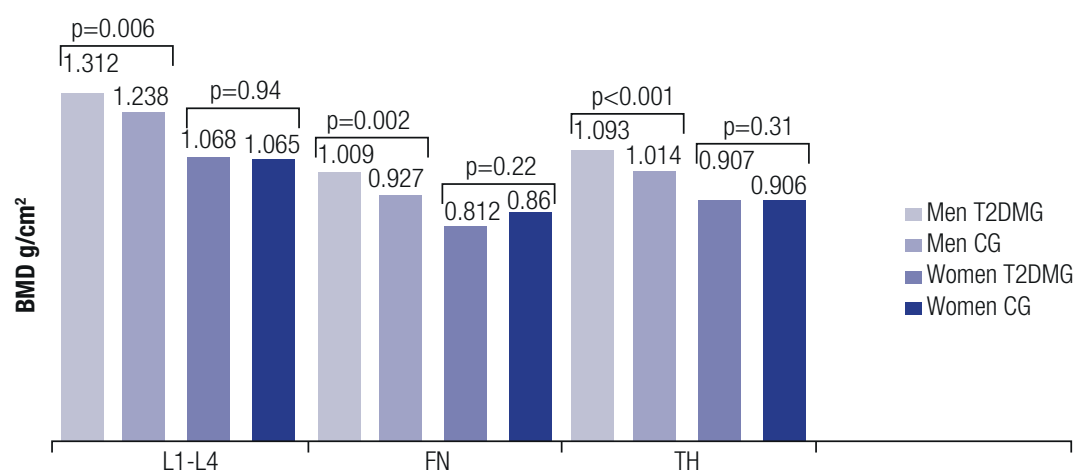

Figure 3. Bone mineral density in both groups.

BMD: bone mineral density; T2DMG: type 2 diabetes mellitus group; CG: control group; FN: femoral neck; TH: total hip; L1-L4: lumbar spine 1 to 4.

Table 2. Variables associated with trabecular bone score on multivariate analysis

\begin{tabular}{lccc}
\hline Predictors & Estimates & 95\% confidence interval & P value \\
\hline Age & -0.004 & -0.005 to -0.002 & $<0.001$ \\
Increased waist circumference & -0.065 & -0.104 to -0.026 & 0.001 \\
Fractures & -0.046 & -0.078 to -0.015 & 0.004 \\
Total lean mass & -0.008 & -0.012 to -0.004 & $<0.001$ \\
Osteoporosis & -0.050 & -0.095 to -0.004 & 0.032 \\
Male & 0.136 & 0.078 to 0.194 & $<0.001$ \\
Body mass index & 0.005 & 0.003 to 0.007 & $<0.001$ \\
Total hip bone mineral density & 0.116 & 0.019 to 0.212 & 0.019 \\
\hline
\end{tabular}

\section{Sarcopenia and osteosarcopenia}

Sarcopenia was present in more subjects in the T2DMG $(\mathrm{n}=23,12.9 \%)$ than the $\mathrm{CG}(\mathrm{n}=8,5.4 \% ; \mathrm{p}<0.030)$ with no difference according to sex $(\mathrm{p}=0.307)$ or ethnicity ( $\mathrm{p}=0.399)$, while osteosarcopenia was present in $21(11.9 \%)$ participants in the T2DMG and $3(2.14 \%)$ of those in the $\mathrm{CG}(\mathrm{p}=0.010)$. Osteopenia was a more frequent contributor to the diagnosis of osteosarcopenia than osteoporosis, as shown in Figure 4.

In women, osteosarcopenia was more prevalent in the T2DMG ( $\mathrm{n}=19$ of 21$)$ compared with the CG $(\mathrm{n}=0$ of $3 ; \mathrm{p}=0.002)$, while the distribution of 


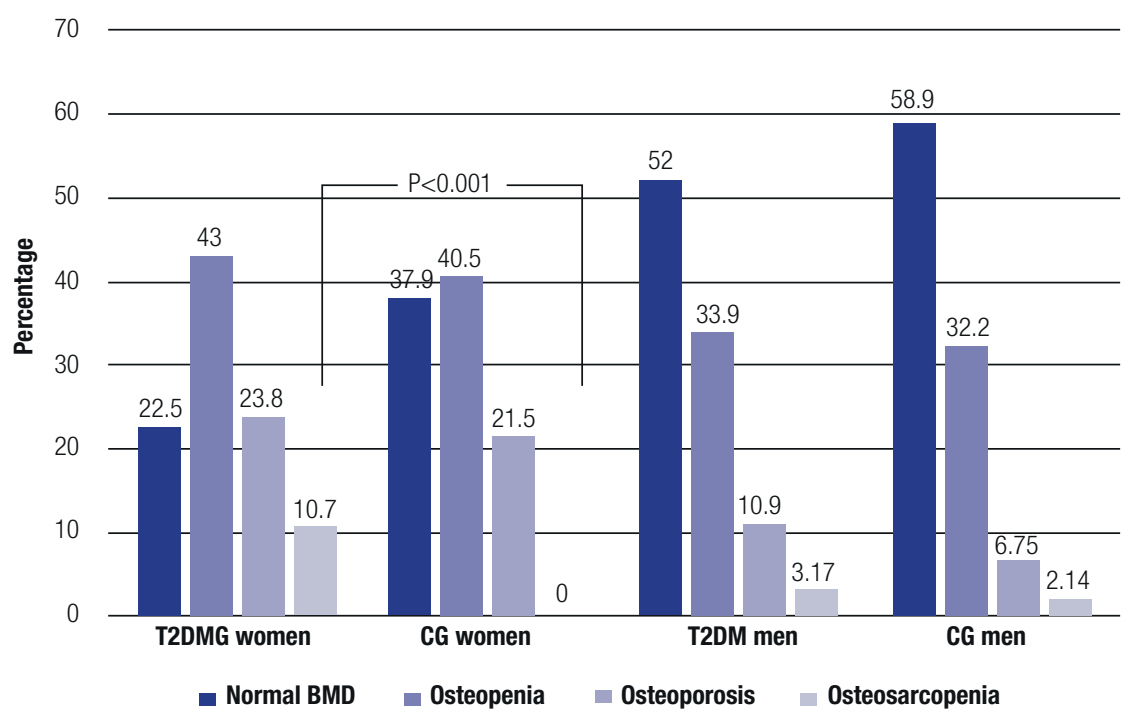

Figure 4. Prevalence of osteopenia, osteoporosis and osteosarcopenia.

T2DMG: type 2 diabetes mellitus group; CG: control group; BMD: bone mineral density.

men with osteosarcopenia was comparable between groups $(\mathrm{p}=0.6870)$ (Figure 4$)$. In the T2DMG, osteosarcopenia was associated with microvascular diabetes complications $(\mathrm{p}=0.03)$, use of calcium and vitamin $\mathrm{D}$ supplements $(\mathrm{p}=0.01)$, and leg fractures $(\mathrm{p}=0.038)$. No association was observed between osteosarcopenia and $\mathrm{HbAlc}$ level $(\mathrm{p}=0.719)$, fasting glucose level $(\mathrm{p}=0.55)$, and disease duration $(\mathrm{p}=$ $0.09)$, or mean TBS value $(\mathrm{p}=0.49)$.

\section{DISCUSSION}

Our study found a higher prevalence of fractures, degraded TBS, sarcopenia, and osteosarcopenia in patients with T2DM compared with controls. The T2DMG comprised patients with long-term diabetes and HbAlc level above the recommended target, both of which have been previously associated with an increased risk of fractures (25).

The prevalence of osteosarcopenia was higher in participants with T2DM compared with controls. In a meta-analysis, rates of osteosarcopenia in elderly individuals varied between $5 \%-37 \%$ (26), but the prevalence of T2DM in the studies was unknown. The presence of osteosarcopenia in the T2DMG in the present study was associated with chronic diabetes complications but not with increased fasting glucose or HbAlc levels. The musculoskeletal unit involves mechanical, physical, and biochemical interactions via paracrine and endocrine communications. A link between the muscle-bone unit and diabetes is osteocalcin, a bone formation marker. Chronic hyperglycemia correlates inversely with circulating osteocalcin in patients with T2DM. Indeed, patients with T2DM have low circulating osteocalcin levels, which may affect bone structure and function (e.g., increased fracture risk), reinforcing the link between skeletal and glucose metabolism (27).

The BMD measurement (the bone component of the osteosarcopenia) indicated a higher frequency of osteopenia compared with osteoporosis and a higher prevalence of osteopenia in women, which is aligned with the literature (28). In patients with T2DM, we found an association between fractures and peripheral neurosensitive motor neuropathy (often accompanied by decreased physical activity and increased falls), which may be a risk factor for osteoporosis, fractures, and sarcopenia (29). Men in the T2DMG had greater BMD than those in the CG, but the same was not found in women. This contrasts with data from the literature (30) and is probably explained by the older age and postmenopausal status of women in our study. Despite these findings, the T2DMG presented the socalled "diabetic paradox," i.e., more frequent fractures despite equal or greater BMD compared with the CG (31). This shows the importance of assessing TBS or other determinants of skeletal strength and fracture risk independent of BMD in patients with T2DM. Studies have shown that TBS is more degraded in patients with T2DM $(32,33)$, which was confirmed in the present 
study. Our study also showed an increased rate of fractures in patients with degraded TBS (32-34).

Possibly due to the small sample size and the crosssectional design of our study, we were unable to verify the association between lower TBS and poor glycemic control reported in other study (35). Degraded bone microarchitecture determined by TBS was present in onethird of our patients and was associated with fractures, osteopenia, and osteoporosis in both sexes, as shown in the literature, but not with osteosarcopenia (36).

Degraded TBS was associated with increased WC, confirming the harmful effect of visceral fat on bone, resulting in low $\mathrm{BMD}$, worse bone microarchitecture, fractures and low TBS (37).

Variabilities in osteosarcopenia rates across different studies are mainly due to variations in the definition of sarcopenia. In the present study, we defined sarcopenia according to the FNIH criteria since these criteria correct the lean mass for body size, fitting better in our sample of overweight and obese patients. Recent initiatives by several international groups have attempted to homogenize the criteria to define sarcopenia, and muscle strength, most often measured by HGS, has become central to the definition. Muscle strength has been recognized as the best predictor of health outcomes in patients with or without diabetes (38). In our study, low HGS was associated with a greater risk of nonvertebral fractures, degraded TBS, and osteosarcopenia, which is aligned with the literature (39). This association may be due to fat infiltration in muscle and bone tissue with consequent osteoporosis and reduced strength; fat infiltration may occur with age or result from chronic inflammation in obesity and T2DM (40).

Limitations of our study include the small sample size, reduced number of men and CG were matched only by age and sex with T2DMG. Also, the study's cross-sectional design does not allow the evaluation of a cause-effect relationship. Strong points of the study are the inclusion of a control group, use of DXA to assess $\mathrm{BC}$, measurement of HGS in all patients and controls, and analysis of fractures and osteosarcopenia in both T2DMG and CG.

In conclusion, patients with T2DM had a higher prevalence of osteosarcopenia and degraded TBS compared with controls. Osteosarcopenia was associated with diabetes complications but not with diabetes duration or glycemic control.
Population aging is increasing the need for care. Prevention, early detection, and better treatment of sarcopenia and osteoporosis in patients with T2DM will be necessary to meet this population's demands in the future.

Acknowledgment: we thank Milena Braga-Basaria, MD (Voxmed Medical Communications) for critically reviewing and suggesting improvements to the manuscript.

Funding: not applicable

Availability of data and materials: the datasets used and/or analyzed during the current study are available from the corresponding author on reasonable request.

Code availability: not applicable

Authors' contributions: LMP collected, analyzed, and interpreted the patient data, collected the control group data and wrote the manuscript; RRP did all the statistical analyses; CAM contributed to the paper and writing corrections; VZCB conceived the study, corrected the paper, and was a major contributor in writing the manuscript.

Ethics approval: this study was approved by the Ethics Committee of Hospital das Clinicas da Universidade Federal do Paraná under the Number 53569116.7.0000.0096. This study was approved by the institutional review board and was performed in accordance with the ethical standards defined in the 1964 Declaration of Helsinki and its amendments.

Consent for publication: All authors read and approved the version to be published and all subsequent versions.

Disclosure: no potential conflict of interest relevant to this article was reported.

\section{REFERENCES}

1. Qi H, Sheng Y, Chen S, Wang S, Zhang A, Cai J, et al. Bone mineral density and trabecular bone score in Chinese subjects with sarcopenia. Aging Clin Exp Res. 2019;31(11):1549-56.

2. Nordklint AK, Almdal TP, Vestergaard P, Lundby-Christensen $L$, Boesgaard TW, Breum L, et al. The effect of metformin versus placebo in combination with insulin analogues on bone mineral density and trabecular bone score in patients with type 2 diabetes mellitus: a randomized placebo-controlled trial. Osteoporos Int. 2018;29(11):2517-26.

3. Oei L, Zillikens MC, Dehghan A, Buitendijk GH, CastañoBetancourt MC, Estrada K, et al. High Bone Mineral Density and Fracture Risk in Type 2 Diabetes as Skeletal Complications of Inadequate Glucose Control: the Rotterdam Study. Diabetes Care. 2013;36(6):1619-28.

4. Vestergaard P. Discrepancies in bone mineral density and fracture risk in patients with type 1 and type 2 diabetes - a meta-analysis. Osteoporos Int. 2007;18(4):427-44. 
5. Janghorbani M, Van Dam RM, Willett WC, Hu FB. Systematic review of type 1 and type 2 diabetes mellitus and risk of fracture. Am J Epidemiol. 2007;166(5):495-505.

6. Schacter GI, LeslieWD. DXA-Based Measurements in Diabetes: Can They Predict Fracture Risk? CalcifTissue Int. 2017;100(2):150-64.

7. Dhaliwal R, Cibula D, Ghosh C, Weinstock RS, Moses AM. Bone quality assessment in type 2 diabetes mellitus. Osteoporos Int. 2014;25(7):1969-73.

8. Ho-Pham LT, Nguyen TV. Association between trabecular bone score and type 2 diabetes: a quantitative update of evidence. Osteoporos Int. 2019;30(10):2079-85.

9. Silva BC, Leslie WD. Trabecular Bone Score: A New DXA-Derived Measurement for Fracture Risk Assessment. Endocrinol Metab Clin North Am. 2017;46(1):153-80.

10. Veronese N, Pizzol D, Demurtas J, Soysal P, Smith L, Sieber C, et al. Association between sarcopenia and diabetes: a systematic review and meta-analysis of observational studies. Eur Geriatr Med. 2019;10(5):685-96.

11. Dalle S, Koppo K. Is inflammatory signaling involved in diseaserelated muscle wasting? Evidence from osteoarthritis, chronic obstructive pulmonary disease and type II diabetes. Exp Gerontol. 2020;137:110964.

12. Cawthon PM. Recent progress in sarcopenia research: a focus on operationalizing a definition of sarcopenia. Curr Osteoporos Rep. 2018;16(6):730-7.

13. Zanker J, Duque G. Osteosarcopenia: the Path Beyond Controversy. Curr Osteoporos Rep. 2020;18(2):81-4.

14. World Health Organization. Assessment of fracture risk and its application to screening for postmenopausal osteoporosis: report of a WHO study group ["meeting held in Rome from 22 to 25 June 1992]". World Health Organization. (1994). [cited 2021 July 6]. Available from. https://apps.who.int/iris/handle/10665/39142

15. Landi F, Calvani R, Ortolani E, Salini S, Martone AM, Santoro L, et al. The association between sarcopenia and functional outcomes among older patients with hip fracture undergoing in-hospital rehabilitation. Osteoporos Int. 2017;28(5):1569-76.

16. Brasil. Ministério da Saúde. Secretaria de Atenção à Saúde. Departamento de Atenção Básica. Dietary Guidelines for the Brazilian population. $2^{\mathrm{a}}$ ed. Brasília: Ministério da Saúde; 2014.

17. WHO Consultation on Obesity (1999: Geneva, Switzerland) \& World Health Organization. (2000). [Internet] Obesity: preventing and managing the global epidemic: report of a WHO consultation. World Health Organization. [cited 2021 July 6]. Available from: https://apps.who.int/iris/handle/10665/42330

18. Expert Panel on Detection, Evaluation, and Treatment of High Blood Cholesterol in Adults. Executive Summary of The Third Report of The National Cholesterol Education Program (NCEP) Expert Panel on Detection, Evaluation, and Treatment of High Blood Cholesterol in Adults (Adult Treatment Panel III). JAMA. 2001;285(19):2486-97.

19. The International Society for Clinical Densitometry [Internet]. 2019 Position Development Conference Task Forces Middletown [cited July 6 2021]. Available from: https://iscd.org/learn/official-positions/

20. WHO scientific group on the assessment of osteoporosis at primary health care level Summary Meeting Report Brussels, Belgium, 5-7 May 2004

21. Medmaps [Internet]. 2019 [ cited 2021 July 6]. Available from: https://www.medimapsgroup.com/

22. Pechmann LM, Jonasson TH, Canossa VS, Trierweiler $H$, Kisielewicz G, Petterle RR, et al. Sarcopenia in Type 2 Diabetes Mellitus: A Cross-Sectional Observational Study. Int J Endocrinol. 2020;2020:7841390.
23. Holick MF, Binkley NC, Bischoff-Ferrari HA, Gordon CM, Hanley $\mathrm{DA}$, Heaney RP, et al. Guidelines for preventing and treating vitamin $\mathrm{D}$ deficiency and insufficiency revisited. J Clin Endocrinol Metab. 2012;97(4):1153-8.

24. Sociedade Brasileira de Diabetes. Diretrizes da Sociedade Brasileira de Diabetes 2017-2018. São Paulo: Clannad; 2017.

25. Majumdar SR, Leslie WD, Lix LM, Morin SN, Johansson H, Oden A, et al. Longer Duration of Diabetes Strongly Impacts Fracture Risk Assessment: The Manitoba BMD Cohort. J Clin Endocrinol Metab. 2016;101(11):4489-96.

26. Nielsen BR, Abdulla J, Andersen HE, Schwarz P, Suetta C. Sarcopenia and osteoporosis in older people: a systematic review and meta-analysis. Eur Geriatr Med. 2018;9(4):419-34.

27. Bilotta FL, Arcidiacono B, Messineo S, Greco M, Chiefari E, Britti $D$, et al. Insulin and osteocalcin: further evidence for a mutual cross-talk. Endocrine. 2018;59(3):622-32.

28. Hirschfeld HP, Kinsella R, Duque G. Osteosarcopenia: where bone, muscle, and fat collide. Osteoporos Int. 2017;28(10):2781-90.

29. Sheu $Y$, Marshall LM, Holton KF, Caserotti $P$, Boudreau RM, Strotmeyer ES, et al. Abdominal body composition measured by quantitative computed tomography and risk of non-spine fractures: the Osteoporotic Fractures in Men (MrOS) Study. Osteoporos Int. 2013;24(8):2231-41.

30. Leslie WD, Aubry-Rozier B, Lamy O, Hans D; Manitoba Bone Density Program. TBS (trabecular bone score) and diabetesrelated fracture risk. J Clin Endocrinol Metab. 2013;98(2):602-9.

31. Walsh JS, Vilaca T. Obesity, Type 2 Diabetes and Bone in Adults. CalcifTissue Int. 2017;100(5):528-35.

32. McCloskey EV, Odén A, Harvey NC, Leslie WD, Hans D, Johansson $\mathrm{H}$, et al. A Meta-Analysis of Trabecular Bone Score in Fracture Risk Prediction and Its Relationship to FRAX. J Bone Miner Res. 2016;31(5):940-8.

33. Hans D, Goertzen AL, Krieg MA, LeslieWD. Bone microarchitecture assessed by TBS predicts osteoporotic fractures independent of bone density: the Manitoba study. J Bone Miner Res. 2011;26(11):2762-9.

34. Leslie WD, Johansson H, McCloskey EV, Harvey NC, Kanis JA, Hans D. Comparison of Methods for Improving Fracture Risk Assessment in Diabetes: The Manitoba BMD Registry. J Bone Miner Res. 2018; 33(11):1923-30.

35. Kim JH, Choi HJ, Ku EJ, Kim KM, Kim SW, Cho NH, et al. Trabecular bone score as an indicator for skeletal deterioration in diabetes. J Clin Endocrinol Metab. 2015;100(2):475-82.

36. Ng AC, Melton LJ 3rd, Atkinson EJ, Achenbach SJ, Holets MF, Peterson JM, et al. Relationship of adiposity to bone volumetric density and microstructure in men and women across the adult lifespan. Bone; 2013;55(1):119-25.

37. Moon HU, Lee N, Chung YS, Choi YJ. Reduction of visceral fat could be related to the improvement of TBS in diabetes mellitus. J Bone Miner Res. 2020;38(5):702-9.

38. Bhasin S, Travison TG, Manini TM, Patel S, Pencina KM, Fielding RA, et al. Sarcopenia Definition: The Position Statements of the Sarcopenia Definition and Outcomes Consortium. J Am Geriatr Soc. 2020;68(7):1410-8.

39. Kamiya K, Kajita E, Tachiki T, Ikehara S, Kouda K, Sato Y, et al. Association between hand-grip strength and site-specific risks of major osteoporotic fracture: Results from the Japanese Population-based Osteoporosis Cohort Study. Maturitas. 2019;130:13-20.

40. Sarodnik C, Bours S, Schaper NC, van den Bergh JP, van Geel T. The risks of sarcopenia, falls and fractures in patients with type 2 diabetes mellitus. Maturitas. 2020;109:70-7. 
Supplementary Table. Variables associated with low or normal trabecular bone score in patients with type 2 diabetes mellitus

\begin{tabular}{|c|c|c|c|}
\hline Variables & Low TBS & Normal TBS & $P$ value \\
\hline \multicolumn{4}{|l|}{ Women } \\
\hline BMI $\left(\mathrm{kg} / \mathrm{m}^{2}\right)$ & $8.5 \pm 4.7$ & $30.8 \pm 5.5$ & 0.064 \\
\hline WC (cm) & $96.7 \pm 12.4$ & $100.5 \pm 11.01$ & 0.154 \\
\hline $\mathrm{HbA1c}$ & $7.95 \pm 1.8$ & $8.08 \pm 1.5$ & 0.526 \\
\hline Glycemia (mg/dL) & $133 \pm 61$ & $147 \pm 91$ & 0.25 \\
\hline PTH (pg/mL) & $78.5 \pm 52.7$ & $50.2 \pm 24.7$ & 0.018 \\
\hline Calcium (mg/dL) & $9.4 \pm 1.1$ & $9.3 \pm 0.8$ & 0.547 \\
\hline $\begin{array}{l}\text { Calcium and vitamin D } \\
\text { supplementation }\end{array}$ & $42(57.5 \%)$ & $8(28.5 \%)$ & 0.017 \\
\hline History of fracture & 26 (35.6\%) & $3(10.7 \%)$ & 0.014 \\
\hline \multicolumn{4}{|l|}{$\mathrm{BMD}\left(\mathrm{g} / \mathrm{cm}^{2}\right)$} \\
\hline Lumbar spine & $1.037 \pm 0.17$ & $1.172 \pm 0.16$ & 0.001 \\
\hline Femoral neck & $0.762 \pm 0.5$ & $0.938 \pm 0.13$ & $<0.001$ \\
\hline Total hip & $0.901 \pm 0.16$ & $1.031 \pm 0.12$ & $<0.001$ \\
\hline Osteopenia & 37 (50.6\%) & $12(46.1 \%)$ & 0.866 \\
\hline Osteoporosis & 20 (27.3\%) & $1(3.84 \%)$ & 0.011 \\
\hline ALM (kg) & $17.1 \pm 4.7$ & $17.6 \pm 2.9$ & 0.366 \\
\hline Low muscle mass & 7 (26.9\%) & $16(22.5 \%)$ & 0.788 \\
\hline HGS (kg) & $19.4 \pm 6.0$ & $19.6 \pm 5.9$ & 0.858 \\
\hline $\mathrm{GS}(\mathrm{m} / \mathrm{s})$ & $1.030 \pm 0.29$ & $1.132 \pm 0.32$ & 0.288 \\
\hline \multicolumn{4}{|l|}{ Men } \\
\hline BMI $\left(\mathrm{kg} / \mathrm{m}^{2}\right)$ & $29.4 \pm 4.04$ & $28.08 \pm 4.48$ & 0.220 \\
\hline WC (cm) & $100.8 \pm 11.0$ & $103.4 \pm 9.1$ & 0.383 \\
\hline $\mathrm{HbA1c}$ & $8.45 \pm 1.6$ & $8.04 \pm 1.7$ & 0.373 \\
\hline Glycemia (mg/dL) & $181 \pm 73$ & $150 \pm 54$ & 0.06 \\
\hline Calcium (mg/dL) & $9.3 \pm 0.45$ & $9.3 \pm 0.67$ & 0.981 \\
\hline Cataract & $5(22.7 \%)$ & $1(2.94 \%)$ & 0.029 \\
\hline History of fracture & $11(50 \%)$ & $7(20.5 \%)$ & 0.044 \\
\hline \multicolumn{4}{|l|}{$\operatorname{BMD}\left(\mathrm{g} / \mathrm{cm}^{2}\right)$} \\
\hline Lumbar spine & $1.227 \pm 1.20$ & $1.368 \pm 1.3$ & 0.013 \\
\hline Femoral neck & $0.917 \pm 0.15$ & $1.046 \pm 0.12$ & 0.002 \\
\hline Total hip & $0.982 \pm 0.25$ & $1.149 \pm 0.12$ & 0.001 \\
\hline Osteopenia & $12(57.1 \%)$ & $8(52.9 \%)$ & 0.025 \\
\hline Osteoporosis & 3 (14.2\%) & 1 (2.94\%) & 0.150 \\
\hline ALM (kg) & $23.3 \pm 2.5$ & $26.2 \pm 8.9$ & 0.305 \\
\hline Low muscle mass & 7 (33.3\%) & 10 (29.4\%) & 0.772 \\
\hline HGS (kg) & $32.2 \pm 13.77$ & $34.45 \pm 6.88$ & 0.081 \\
\hline $\mathrm{GS}(\mathrm{m} / \mathrm{s})$ & $1.32 \pm 0.29$ & $1.28 \pm 0.34$ & 0.736 \\
\hline
\end{tabular}

Data are presented as mean \pm standard deviation or frequency (percentage). BMI: body mass index; WC: waist circumference; PTH: parathyroid hormone; HGS: handgrip strength; GS: gait speed; BMD: bone mineral density; ALM: appendicular lean mass. Low muscle mass was defined as ALM/BMl $<0.789$ in men and $<0.512$ in women. 